\title{
Bayesian Multilateration
}

This paper was downloaded from TechRxiv (https://www.techrxiv.org).

\section{LICENSE}

CC BY 4.0

SUBMISSION DATE / POSTED DATE

$13-10-2021 / 29-10-2021$

CITATION

Alencar, Alisson; Mattos, César; Gomes, João; Mesquita, Diego (2021): Bayesian Multilateration. TechRxiv. Preprint. https://doi.org/10.36227/techrxiv.16806688.v1

$\mathrm{DOI}$

10.36227/techrxiv.16806688.v1 


\title{
Bayesian Multilateration
}

\author{
Alisson S. C. Alencar, César L. C. Mattos, João P. P. Gomes, Diego Mesquita
}

\begin{abstract}
Multilateration (MLAT) is the de facto standard to localize points of interest (POIs) in navigation and surveillance systems. Despite sensors being inherently noisy, most existing techniques i) are oblivious to noise patterns in sensor measurements; and ii) only provide point estimates of the POI's location. This often results in unreliable estimates with high variance, i.e., that are highly sensitive to measurement noise. To overcome this caveat, we advocate the use of Bayesian modeling. Using Bayesian statistics, we provide a comprehensive guide to handle uncertainties in MLAT. We provide principled choices for the likelihood function and the prior distributions. Inference within the resulting model follows standard MCMC techniques. Besides coping with unreliable measurements, our framework can also deal with sensors whose location is not completely known, which is an asset in mobile systems. The proposed solution also naturally incorporates multiple measurements per reference point, a common practical situation that is usually not handled directly by other approaches. Comprehensive experiments with both synthetic and real-world data indicate that our Bayesian approach to the MLAT task provides better position estimation and uncertainty quantification when compared to the available alternatives.
\end{abstract}

Index Terms-Multilateration, Bayesian modeling, Localization, Navigation

\section{INTRODUCTION}

A CCURATELY estimating a target's position is fundamental in many real-world applications, such as wireless sensor networks [17], airport surveillance [9], and navigation of unmanned vehicles or robots [18]. In environments that limit the use of radar technologies and GPS, localization based on Multilateration (MLAT) is a widely used solution [10].

Multilateration is a well-known localization method that leverages distances from multiple reference points to find the position of a Point Of Interest (POI). If i) we know the location of reference points exactly and ii) measurements are noiseless, these distances allow the MLAT algorithm localizes the POI.

Over the years, several MLAT solutions have been proposed with the implicit assumption that all the involved quantities are known exactly [5], [16], [13], [2]. Although these solutions may be useful in some situations, in many real scenarios the distances to the reference points and the position of the reference points themselves may be uncertain. When this is the case, a probabilistic approach can offer a more comprehensive solution, accounting for both the uncertainty around the reference points and the distances.

Menchaca-Mendez et al. [11] use normal distributions to model the uncertainty in the position of each reference point. Then, they estimate the POI's position via maximum likelihood, finding the mode of a product of normals related

AA (alencar.alisson@ifce.edu.br), CM (cesarlincoln@dc.ufc.br) and JG (jpaulo@dc.ufc.br) are with the Federal University of Ceará. AA is also with the Federal Institute of Ceará. DM (diego.mesquita@aalto.fi) is with the Aalto University.

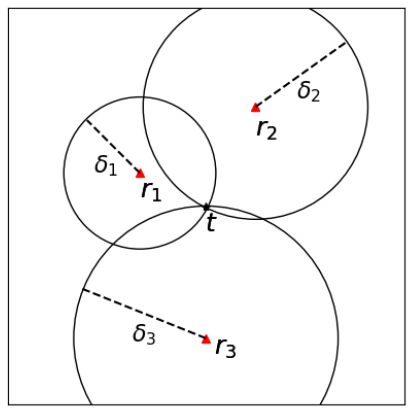

(a)

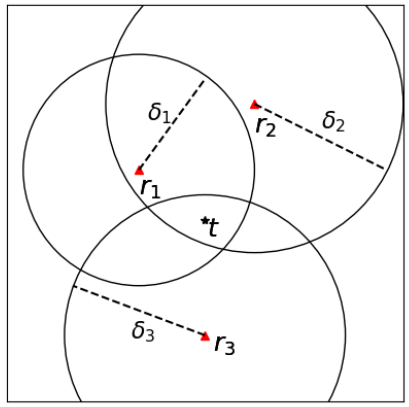

(b)
Fig. 1: Noise matters. (a) When all measurements are accurately obtained, the POI lies exactly in the intersection of the circumferences centered on the reference points with a radius equal to the observed distances; (b) When there is uncertainty in the measurements, the POI is positioned elsewhere and cannot be uniquely determined.

to each reference point. In contrast, Vasconcelos et al. [6], assume the locations of reference points are known, but the distances and the position of the POI are uncertain. They use Rayleigh likelihoods to factor in the distances and use a normal distribution as prior for the POI position. Despite using a Bayesian approach to marginalize the unknown quantities, their experimental evaluation considers only point estimates of the POI's location.

None of the aforementioned works explores the scenario where both the distances and the positions of the reference points are uncertain. Moreover, these approaches evaluate only point estimates of the POI's position, totally ignoring the uncertainty around predictions.

We present a fully Bayesian formulation of the multilateration problem. Our method, Bayesian Multilateration (BMLAT) accounts for the uncertainties in all variables of interest (i.e., reference point positions, distances between the POI and reference points, and the POI's position). We develop a novel parameterization of the Nakagami distribution, that we use as likelihood in BMLAT. We also briefly discuss how to incorporate knowledge through priors. Furthermore, our probabilistic formulation inherently handles scenarios where multiple noisy distance measurements are available for each reference point, a common practical situation. Importantly, BMLAT is easy to implement and we can assess its full posterior using off-theshelf Markov Chain Monte Carlo (MCMC) software [14].

Since the proposed BMLAT is flexible enough to incorporate knowledge from a given application of interest and inference follows standard Bayesian procedures, we expect it to become a valuable tool for practitioners. 


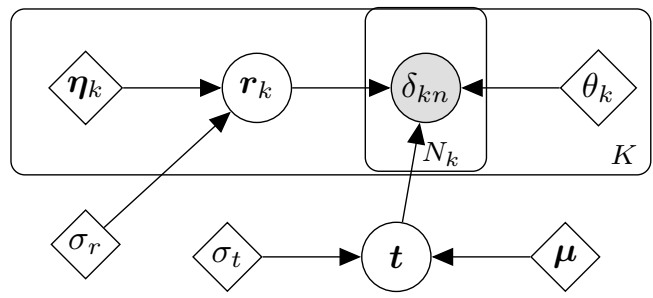

Fig. 2: Graphical model for BMLAT. Diamonds denote hyperparameters, circles are random variables, and shaded nodes are observations. The plate indicates repetition.

\section{Multilateration}

Suppose $D$ is the dimension of the euclidean space we are working on ${ }^{1}$. Let $t \in \mathbb{R}^{D}$ be the POI position, $\boldsymbol{r}_{k} \in \mathbb{R}^{D}$ the position of the $k$-th reference point, $K$ the number of reference points, $N_{k}$ the number of observed distances for the $k$-th reference point, $\delta_{k n}$ the $n$-th observed distance for the $k$-th reference point, and $\boldsymbol{\delta}=\left\{\delta_{k n}, \forall k, n \in \mathbb{Z}^{+} \mid k \leq K\right.$ and $\left.n \leq N_{k}\right\}$ the set of observed distances.

Multilateration is a localization task based on the observations of distances to reference points. Fig. 1 illustrates a scenario with $K=3$ reference points in $\mathbb{R}^{2}$. In (a) the measurements are known exactly, while in (b) there is uncertainty in the measurements. When the observations are accurate, the POI is exactly at the intersection of the circles centered on the reference points, with each radius given by the observed distances. However, this is not true when there is inaccuracy in the distance measurements. The most common way to deal with such an issue is to look for the point whose distances to the reference points are as close as possible to the observed distances, a task that can be modeled as a non-linear optimization problem.

In the following section, we use Bayesian statistics to tackle the MLAT task. We incorporate all uncertainties using probability distributions and then use the Bayes' rule to update our belief about the POI's position.

\section{BAYESIAN MUltilateration}

The graphical model of the proposed Bayesian formulation for the MLAT task is depicted in Fig. 2. Our goal is to compute the posterior probability $p(\boldsymbol{t} \mid \boldsymbol{\delta})$ given the set $\boldsymbol{\delta}$ of observed distances for each reference point. The problem formulation is mathematically described by

$$
p(\boldsymbol{t} \mid \boldsymbol{\delta}) \propto p(\boldsymbol{t}) \prod_{k=1}^{K} \int \prod_{n=1}^{N_{k}} p\left(\delta_{k n} \mid \boldsymbol{t}, \boldsymbol{r}_{k}\right) p\left(\boldsymbol{r}_{k}\right) \mathrm{d} \boldsymbol{r}_{k},
$$

where $p(\boldsymbol{t})$ and $p\left(\boldsymbol{r}_{k}\right)$ are respectively the priors for the POI and the reference point $\boldsymbol{r}_{k}$, and $p\left(\delta_{k n} \mid \boldsymbol{t}, \boldsymbol{r}_{k}\right)$ is the likelihood function. Their choices are described as follows.

\footnotetext{
${ }^{1}$ We consider only $D=2$ and leave scenarios w/ $D>2$ to further work.
}

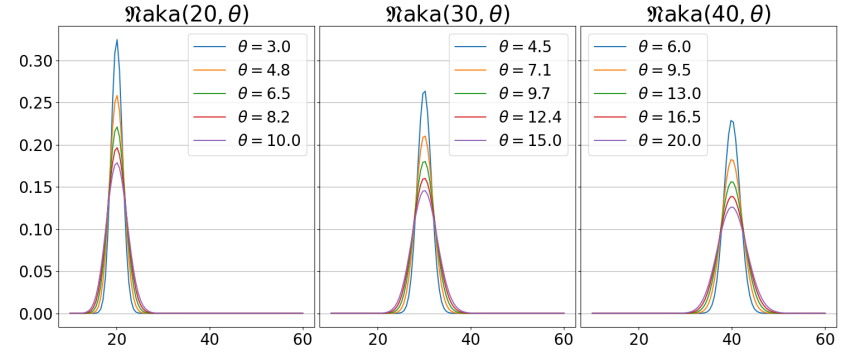

Fig. 3: Reparameterized Nakagami. Influence of the parameter $\theta$ on the shape of the likelihood. All distributions above have mode equal to $\delta$, but become flatter as $\theta$ increases.

\section{A. Prior Distributions}

We need to define prior distributions for the POI and the reference points. In both cases, in the absence of additional knowledge, we opt for distributions that cover the entire $\mathbb{R}^{D}$. Specifically, we use multivariate Gaussians with diagonal covariances. Let $\mathbb{I}_{D}$ denote the $D$-dimensional identity matrix, the priors are given by

$$
\begin{aligned}
\boldsymbol{t} & \sim \mathcal{N}\left(\boldsymbol{\mu}, \sigma_{t}^{2} \mathbb{I}_{D}\right), \\
\boldsymbol{r}_{\boldsymbol{k}} & \sim \mathcal{N}\left(\boldsymbol{\eta}_{\boldsymbol{k}}, \sigma_{r}^{2} \mathbb{I}_{D}\right) .
\end{aligned}
$$

We can easily incorporate prior knowledge by setting the hyper-parameters in Equation 2. The value $\boldsymbol{\mu} \in \mathbb{R}^{D}$ can be an initial guess for the position $t$. It can be obtained, for instance, using some conventional MLAT solver. $\sigma_{t}>0$ quantifies the uncertainty about this initial guess and a high value can be used to indicate an uninformative prior. The $\eta_{k} \in \mathbb{R}^{D}, \forall k$, values can be the measured reference points positions, the same used in standard MLAT approaches. Finally, $\sigma_{r}>0$ indicates the uncertainty regarding such positions.

\section{B. Likelihood Function}

Since $\delta_{k n}$ represents the $n$-th measured distance from the $k$ th reference point, we must choose a likelihood function such as that $p\left(\delta_{k n} \mid \boldsymbol{t}, \boldsymbol{r}_{\boldsymbol{k}}\right)=0, \forall \delta_{k n}<0$. Thus, only likelihoods with positive support are suitable. Squared distances between random variables with different distributions can be approximated by a gamma distribution [3], [15], [7], [1]. By taking the square root of a gamma random variable, a Nakagami random variable is obtained. Thus, in our framework, we choose a Nakagami likelihood to represent the measured distances. A similar assumption was also presented in [12].

The Nakagami distribution is described in terms of the shape parameter $m \geq \frac{1}{2}$ and the spread parameter $\Omega>0$ :

$$
\operatorname{Naka}(x \mid m, \Omega)=\frac{2 m^{m}}{\Gamma(m) \Omega^{m}} x^{2 m-1} e^{-\frac{m}{\Omega} x^{2}} .
$$

We choose those parameters so that the distribution mode is at the calculated distance $\hat{\delta}_{k}=\left\|\boldsymbol{t}-\boldsymbol{r}_{\boldsymbol{k}}\right\|$ to the corresponding reference point. The spread constitutes a free hyperparameter of the model. Thus, for the $k$-th reference point:

$$
\hat{\delta}_{k}=\overbrace{\frac{\sqrt{2}}{2} \sqrt{\frac{\left(2 m_{k}-1\right) \Omega_{k}}{m_{k}}}}^{\text {mode of Naka }\left(m_{k}, \Omega_{k}\right)} \Longrightarrow m_{k}=\frac{\Omega_{k}}{2\left(\Omega_{k}-\hat{\delta}_{k}^{2}\right)} .
$$




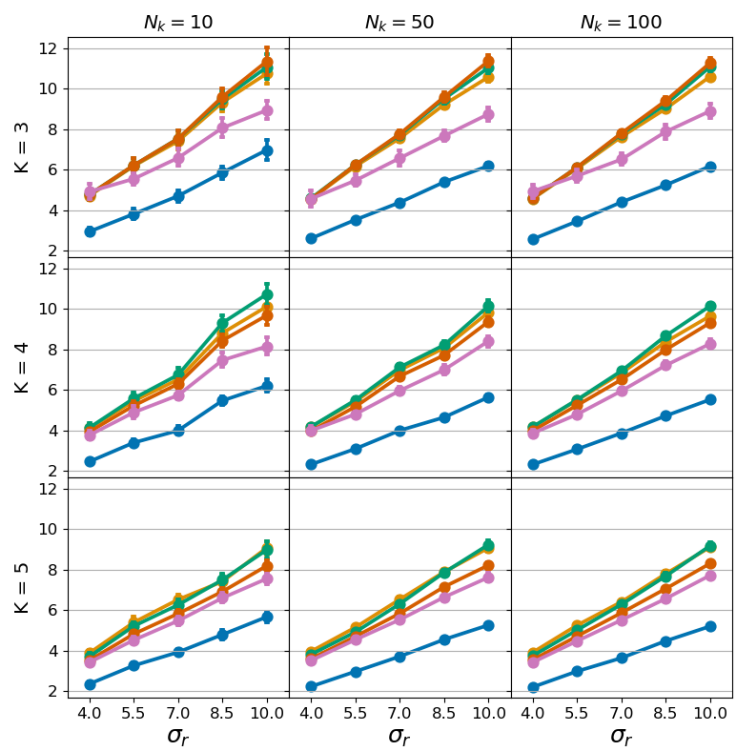

(a) RMSE w.r.t. POI position.

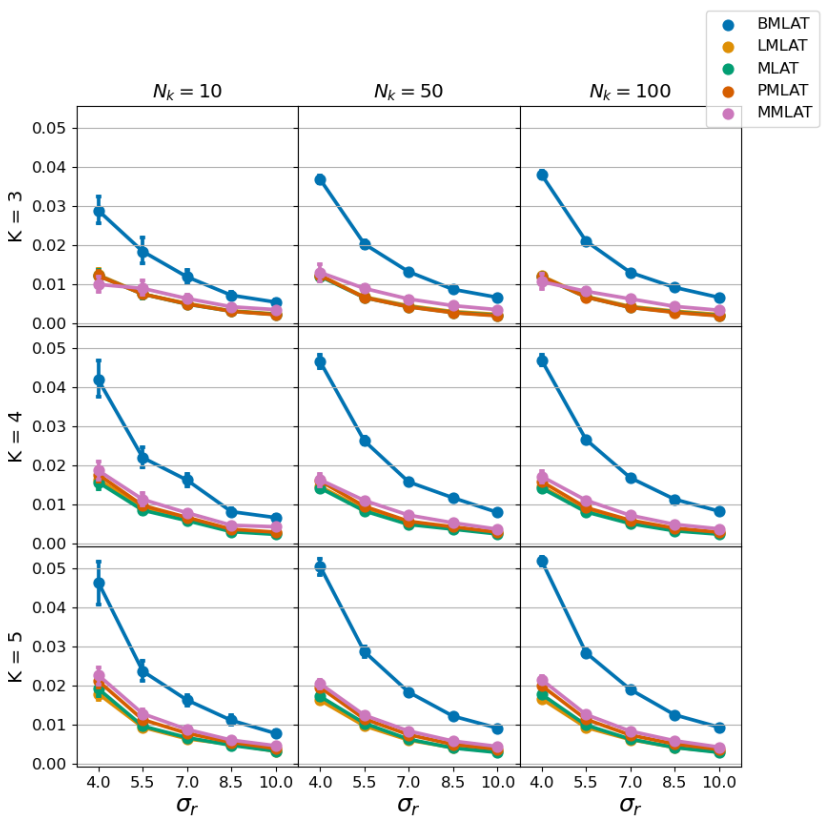

(b) Posterior density of the POI.

Fig. 4: Results for the artificial dataset. (a) In terms of RMSE, BMLAT outperforms the other methods for any number of references and observation. (b) Additionally, BMLAT is the method that places higher density on the true POI value.

In the above formulation, we need to ensure that $\Omega_{k}>\hat{\delta}_{k}^{2}$. Thus, we introduce the hyperparameter $\theta_{k}$ and define $\Omega_{k}=$ $\theta_{k}+\hat{\delta}_{k}^{2}$. This ensures that the previous constraint will be met for every $\theta_{k}>0$. Furthermore, by substituting the value of $\theta_{k}$ in Eq. (4), we conclude that $m_{k}=\frac{\theta_{k}+\hat{\delta}_{k}^{2}}{2 \theta_{k}}$. From this definition, the likelihood function depends only on the calculated distance $\hat{\delta}_{k}$ and the spread parameter $\theta_{k}$. Thus, for simplicity, we define a reparameterization of the Nakagami distribution denoted by Naka. For any $\delta \geq 0$ and any positive $\theta$, it is written as:

$$
\operatorname{Naka}(\delta, \theta) \triangleq \operatorname{Naka}\left(m=\frac{\theta+\delta^{2}}{2 \theta}, \Omega=\theta+\delta^{2}\right) .
$$

To illustrate the influence of $\theta$, we evaluate the Nakagami likelihood function according to the above parameterization considering the calculated distances $\delta \in\{20,30,40\}$ and $\frac{3 \delta}{20} \leq \theta \leq \frac{10 \delta}{20}$. The results can be seen in Fig. 3. Regardless of the $\theta$ value, the mode remains at the calculated distance $\delta$. Moreover, the larger the $\theta$, the wider the curve of the likelihood. Furthermore, one can show experimentally that there is a linear relation between $\theta$ and the variance of the likelihood, given by $\operatorname{var}[\mathfrak{N a k a}(\delta, \theta)] \approx \frac{\theta}{2}$.

The final reparametrized likelihood function is given by

$$
\delta_{k n} \sim \operatorname{Naka}\left(\left\|\boldsymbol{t}-\boldsymbol{r}_{\boldsymbol{k}}\right\|, \theta_{k}\right) .
$$

\section{Bayesian inference}

The choices that we have made for the priors and the likelihood function do not result in a conjugate model for the Eq. (1). Thus, we follow a MCMC strategy to sample from the posterior $p(\boldsymbol{t} \mid \boldsymbol{\delta})$ of the POI given the measured distances. In this work, we use the NUTS algorithm [4] as implemented in the Python library pyStan [14]. The use of other approximate inference methods is left for future investigations.

\section{EXPERIMENTS}

We evaluate our proposal using both real and artificial datasets. We use MLAT [10], LMLAT [5], PMLAT [11], and MMLAT [6] as baselines. For a fair comparison, we use bootstrap to derive uncertainty estimates for these methods. More specifically, we run each classical method 2000 times, each one with a random measure of one among the $N_{k}$ distances to each reference point. We emphasize that the proposed BMLAT can directly cope with all the available measurements and compute a posterior distribution for the POI. In all experiments, we use the result of the linear method (LMLAT) to define the parameters of the POI's prior distribution, where $\boldsymbol{\mu}$ is the sample mean and $\sigma_{t}^{2}$ is the scale of the covariance matrix.

\section{A. Synthetic data}

Data. The data was generated according to the generative model described in Section III, with $\boldsymbol{\mu}=\mathbf{0}, \sigma_{t}=10$, and $\theta_{k}=$ $8 \forall k$. We fix $\boldsymbol{\eta}_{k}$ at a distance 40 from the POI and distribute it around the POI, so that the distance between two adjacent points is constant. We vary the number of reference points ( $K \in\{3,4,5\})$; the number of distances to each reference point $\left(N_{k} \in\{10,50,100\}, \forall k\right)$; and the standard deviation of the reference points distribution $\left(\sigma_{r} \in\{4,5.5,7,8.5,10\}\right)$.

Results. Fig. 4 shows the mean and the $95 \%$ confidence interval for the RMSE and the posterior density of the POI, along with 30 runs for each method and each scenario. We can see that both metrics have monotonic behaviour about the increase of $\boldsymbol{\sigma}_{r}$. The results also slightly improve as $K$ or $N_{k}$ increase (mainly in the first two columns). In addition, BMLAT consistently obtains better results in all settings. 


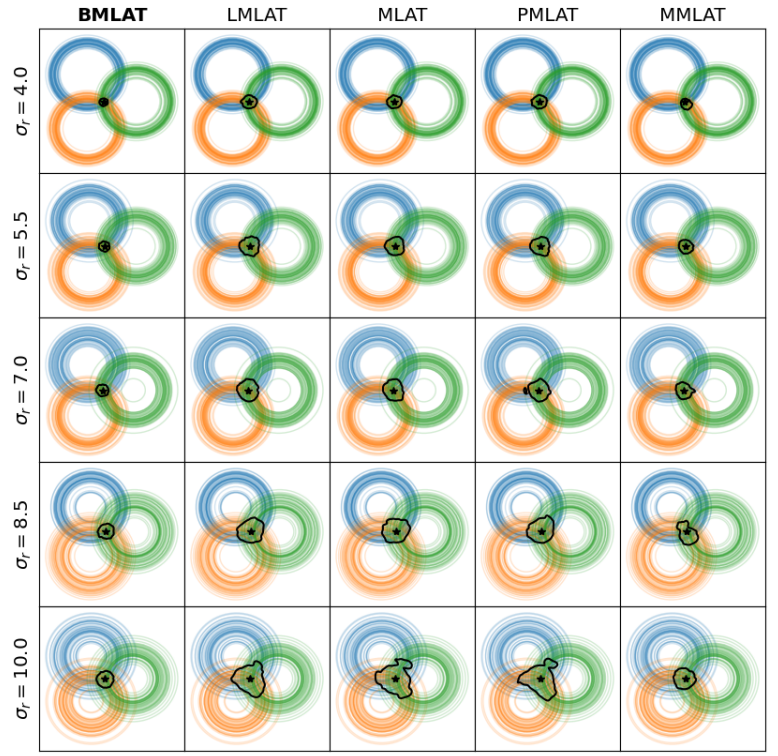

(a) Synthetic data scenarios.

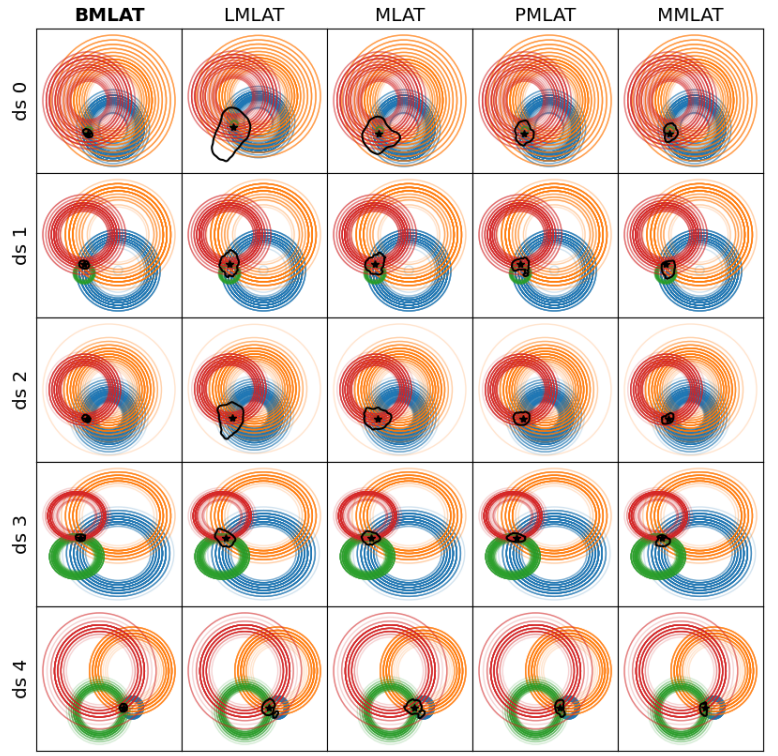

(b) Real data scenarios.

Fig. 5: Does BMLAT concentrate on the right spot? The plots show posterior contours for BMLAT (black curve) for synthetic and real-world data. Colored circles represent distance observations, with different colors for each reference point. The star denotes the true POI location. (a) Shows the effect of increasing the variance around each reference's position. (b) Shows results for five real-world problems. In all cases, the BMLAT posterior concentrates more tightly on the true POI.

\begin{tabular}{|c|c|c|c|c|c|c|c|c|c|c|}
\hline & \multicolumn{5}{|c|}{ Distance / RMSE } & \multicolumn{5}{|c|}{ Posterior density } \\
\hline dataset & BMLAT & LMLAT & MLAT & PMLAT & MMLAT & BMLAT & LMLAT & MLAT & PMLAT & MMLAT \\
\hline $\mathbf{0}$ & $2.02 \mathrm{e}+00$ & $1.06 \mathrm{e}+01$ & $8.58 \mathrm{e}+00$ & $5.26 e+00$ & $4.03 \mathrm{e}+00$ & $6.53 \mathrm{e}-02$ & $2.37 \mathrm{e}-03$ & $3.58 \mathrm{e}-03$ & $1.01 \mathrm{e}-02$ & $1.62 \mathrm{e}-02$ \\
\hline 1 & $1.51 \mathrm{e}+00$ & $3.56 \mathrm{e}+00$ & $3.14 \mathrm{e}+00$ & $2.62 \mathrm{e}+00$ & $3.97 \mathrm{e}+00$ & $1.11 \mathrm{e}-01$ & $1.96 \mathrm{e}-02$ & $2.44 \mathrm{e}-02$ & $3.37 \mathrm{e}-02$ & $1.13 e-02$ \\
\hline 2 & $1.84 e+00$ & $6.86 e+00$ & $5.40 e+00$ & $3.77 e+00$ & $2.74 \mathrm{e}+00$ & $7.58 \mathrm{e}-02$ & $4.81 \mathrm{e}-03$ & $8.42 \mathrm{e}-03$ & $1.81 \mathrm{e}-02$ & $3.67 \mathrm{e}-02$ \\
\hline 3 & $1.18 \mathrm{e}+00$ & $2.76 \mathrm{e}+00$ & $2.32 \mathrm{e}+00$ & $2.02 \mathrm{e}+00$ & $2.91 \mathrm{e}+00$ & $1.82 \mathrm{e}-01$ & $3.52 \mathrm{e}-02$ & $4.85 \mathrm{e}-02$ & $7.18 \mathrm{e}-02$ & $1.56 \mathrm{e}-02$ \\
\hline 4 & $1.26 \mathrm{e}+00$ & $2.44 \mathrm{e}+00$ & $2.72 \mathrm{e}+00$ & $2.06 e+00$ & $2.15 \mathrm{e}+00$ & $1.58 \mathrm{e}-01$ & $3.90 \mathrm{e}-02$ & $3.25 \mathrm{e}-02$ & $5.79 \mathrm{e}-02$ & $4.52 \mathrm{e}-02$ \\
\hline mean & $1.56 \mathrm{e}+00$ & $5.25 e+00$ & $4.43 e+00$ & $3.15 \mathrm{e}+00$ & $3.16 \mathrm{e}+00$ & $1.18 \mathrm{e}-01$ & $2.02 \mathrm{e}-02$ & $2.35 \mathrm{e}-02$ & $3.83 \mathrm{e}-02$ & $2.50 \mathrm{e}-02$ \\
\hline
\end{tabular}

TABLE I: Results for the real dataset.Average distance of the posterior samples to the POI for the real datasets. BMLAT obtained the best performance among all methods, with lower RMSE and placing higher density on the true POI location.

Fig. 5a shows the POI posterior distribution for each method considering the scenarios with $K=3$ reference points and $N_{k}=50, \forall k$, observed distances. All methods have posterior samples approximately centred on the POI, but the BMLAT defines a region with a consistently smaller radius.

\section{B. Real world data}

Data. We use a dataset of indoor measurements using WiFi received signal strength indicator (RSSI)[8], which we convert into distances following the methodology by Zhu and Feng [18]. We focus on 5 POIs, each with approximate 400 distances measurements from 4 reference points.

Results. We evaluate our method with $\sigma_{r}=2$ for all datasets and $\theta_{k}$ equals to the variance of the observed distances for the $k$-th reference point. Table I shows that the BMLAT has significantly better RMSE and posterior density than the other methods. In Fig. 5b, we can see that the posterior distribution of the BMLAT delimits a considerably smaller region.

\section{Concluding Remarks}

We propose using Bayesian statistics to model the uncertainties that arise when applying multilateration to real- world settings. Moreover, we follow standard Bayesian inference strategies to marginalize all the unknown quantities and produce a posterior distribution for the POI position. The priors and the likelihood within our framework were carefully chosen to better represent the characteristics of the localization problem. We have also presented practical guidelines for choosing prior hyperparameters. Importantly, our approach naturally incorporates multiple distance observations for each reference point, a common scenario. Remarkably, BMLAT outperforms classical methods on artificial and real-world data.

In the future, we will evaluate the BMLAT in scenarios where $D>2$. Moreover, to enable BMLAT in embedded devices, it is worth investigating variational approximations for the posterior in Eq. (1). These approximations may provide a valuable trade-off between accuracy and computational effort. Finally, the model itself can be enhanced by including priors to its hyperparameters, which, for instance, would allow different noise variances for each reference point. 


\section{REFERENCES}

[1] Shai Covo and Amir Elalouf. A novel single-gamma approximation to the sum of independent gamma variables, and a generalization to infinitely divisible distributions. Electron. J. Statist., 8(1):894-926, 2014.

[2] Amauri Holanda de Souza Junior, Francesco Corona, Yoan Miche, Amaury Lendasse, Guilherme A. Barreto, and Olli Simula. Minimal learning machine: A new distance-based method for supervised learning. In Ignacio Rojas, Gonzalo Joya, and Joan Gabestany, editors, Advances in Computational Intelligence, pages 408-416, Berlin, Heidelberg, 2013. Springer Berlin Heidelberg.

[3] Marc G. Genton. Skew-Elliptical Distributions and Their Applications: A Journey Beyond Normality. Chapman and Hall/CRC, 2004.

[4] Matthew D Hoffman, Andrew Gelman, et al. The No-U-Turn sampler: adaptively setting path lengths in Hamiltonian Monte Carlo. Journal of Machine Learning Research, 15(1):1593-1623, 2014.

[5] Joonas Hämäläinen, Alisson S. C. Alencar, Tommi Kärkkäinen, César L. C. Mattos, Amauri H. Souza Júnior, and João P. P. Gomes. Minimal learning machine: Theoretical results and clustering-based reference point selection. Journal of Machine Learning Research, 21(239):1-29, 2020.

[6] Daniel Jean Rodrigues Vasconcelos, Amauri Holanda de Souza Júnior, and Francesco Corona. Probabilistic multilateration: Model and inference. In 2019 8th Brazilian Conference on Intelligent Systems (BRACIS), pages 675-680, 2019.

[7] N. Johnson, S. Kotz, and N Balakrishnan. Continuous univariate distributions. Wiley, 1995.

[8] Amir Malekpour. Indoor location determination with rssi, Jan 2018.

[9] Ivan A. Mantilla-Gaviria, M. Leonardi, G. Galati, and J. V. BalbastreTejedor. Localization algorithms for multilateration (mlat) systems in airport surface surveillance. Signal, Image and Video Processing, 9:1549-1558, 2015.

[10] Mateo Marcelić, Bruno Sandrić, Jakov Jelenić, and Marko Jurčević. Determining location in lpwan using multilateration. In 2019 2nd International Colloquium on Smart Grid Metrology (SMAGRIMET), pages 1-4, 2019.

[11] Rolando Menchaca-Mendez, Anabel Pineda-Briseño, Edgar Chávez, Ricardo Menchaca-Mendez, Rolando Quintero, and Miguel Torres-Ruiz. A probabilistic approach to location estimation in manets. Ad Hoc \& Sensor Wireless Networks, 28:97-114, 082015.

[12] Diego Mesquita, Joao Paulo Gomes, Amauri Júnior, and Juvêncio Nobre. Euclidean distance estimation in incomplete datasets. Neurocomputing, 248, 122017

[13] Abdelmoumen Norrdine. An algebraic solution to the multilateration problem. 042015.

[14] Allen Riddell, Ari Hartikainen, and Matthew Carter. pystan (3.0.0). PyPI, March 2021.

[15] Charles Roberts and Seymour Geisser. A necessary and sufficient condition for the square of a random variable to be gamma. Biometrika Trust, 53(1/2):275-278, June 1966.

[16] Yue Wang. Linear least squares localization in sensor networks. EURASIP Journal on Wireless Communications and Networking, 2015, 122015.

[17] Xiaozhen Yan, Qinghua Luo, Yipeng Yang, Sicheng Liu, Heping Li, and Cong Hu. Itl-meposa: Improved trilateration localization with minimum uncertainty propagation and optimized selection of anchor nodes for wireless sensor networks. IEEE Access, 7:53136-53146, 2019.

[18] Xiuyan Zhu and Y. Feng. Rssi-based algorithm for indoor localization. Communications and Network, 05:37-42, 2013. 